\title{
Statistical Scalability Analysis of Communication Operations in Distributed Applications
}

\author{
J. S. Vetter, M. O. McCracken
}

This article was submitted to

ACM SIGPLAN Symposium on Principles and Practice of Parallel Programming, Snowbird, UT, June 18- 20, 2000

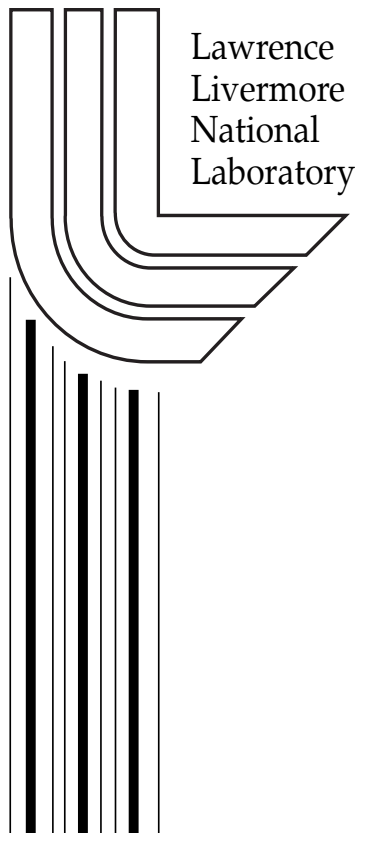

February 27, 2001 


\section{DISCLAIMER}

This document was prepared as an account of work sponsored by an agency of the United States Government. Neither the United States Government nor the University of California nor any of their employees, makes any warranty, express or implied, or assumes any legal liability or responsibility for the accuracy, completeness, or usefulness of any information, apparatus, product, or process disclosed, or represents that its use would not infringe privately owned rights. Reference herein to any specific commercial product, process, or service by trade name, trademark, manufacturer, or otherwise, does not necessarily constitute or imply its endorsement, recommendation, or favoring by the United States Government or the University of California. The views and opinions of authors expressed herein do not necessarily state or reflect those of the United States Government or the University of California, and shall not be used for advertising or product endorsement purposes.

This is a preprint of a paper intended for publication in a journal or proceedings. Since changes may be made before publication, this preprint is made available with the understanding that it will not be cited or reproduced without the permission of the author.

This report has been reproduced directly from the best available copy.

Available electronically at http://www.doc.gov/bridge

Available for a processing fee to U.S. Department of Energy

And its contractors in paper from

U.S. Department of Energy

Office of Scientific and Technical Information

P.O. Box 62

Oak Ridge, TN 37831-0062

Telephone: (865) 576-8401

Facsimile: (865) 576-5728

E-mail: reports@adonis.osti.gov

Available for the sale to the public from

U.S. Department of Commerce

National Technical Information Service

5285 Port Royal Road

Springfield, VA 22161

Telephone: (800) 553-6847

Facsimile: (703) 605-6900

E-mail: orders@ntis.fedworld.gov

Online ordering: http://www.ntis.gov/ordering.htm

OR

Lawrence Livermore National Laboratory Technical Information Department's Digital Library

http://www.llnl.gov/tid/Library.html 


\title{
Statistical Scalability Analysis of Communication Operations in Distributed Applications
}

\author{
Jeffrey S. Vetter \\ Center for Applied Scientific Computing \\ Lawrence Livermore National Laboratory \\ Livermore, California, USA 94551 \\ \{vetter3,mccracken6\}@IInl.gov
}

Michael O. McCracken

\begin{abstract}
Current trends in high performance computing suggest that users will soon have widespread access to clusters of multiprocessors with hundreds, if not thousands, of processors. This unprecedented degree of parallelism will undoubtedly expose scalability limitations in existing applications, where scalability is the ability of a parallel algorithm on a parallel architecture to effectively utilize an increasing number of processors. Users will need precise and automated techniques for detecting the cause of limited scalability. This paper addresses this dilemma. First, we argue that users face numerous challenges in understanding application scalability: managing substantial amounts of experiment data, extracting useful trends from this data, and reconciling performance information with their application's design. Second, we propose a solution to automate this data analysis problem by applying fundamental statistical techniques to scalability experiment data. Finally, we evaluate our operational prototype on several applications, and show that statistical techniques offer an effective strategy for assessing application scalability. In particular, we find that non-parametric correlation of the number of tasks to the ratio of the time for individual communication operations to overall communication time provides a reliable measure for identifying communication operations that scale poorly.
\end{abstract}

\section{INTRODUCTION}

Current trends in high performance computing suggest that users will be running their applications on scalable clusters of multiprocessors with hundreds, if not thousands, of processors in the near future $[3,15]$. This unprecedented availability of computing resources motivates the need for precise and meaningful scalability analysis of these applications. By scalability, we mean the ability of a parallel algorithm on a

Submitted to ACM PPOPP 2001. parallel architecture to effectively utilize an increasing number of processors $[6,7,13]$. Undoubtedly, this new, high degree of concurrency will expose scalability limitations of applications that, at lower levels of concurrency, might have been shrouded by other application or system characteristics. Furthermore, perpetual improvements in single node performance will continue to direct users' attention on the scalability limitations of communication operations in their distributed memory applications.

Although metrics like execution time, speedup, and efficiency [14] help quantify scalability on an abstract level, users need precise information about the communication operations in their application that scale poorly. In addition, for any analysis to help users understand their application's scalability, the technology should be able to explain scalability phenomena in terms of decisions a user makes while designing their application.

To this end, we propose an automated technique that uses familiar statistical techniques to direct a user's attention on poorly scaling communication operations in their application. Our method digests the results of multiple application experiments and suggests communication operations whose growth has a positive correlation with the number of tasks. We empirically evaluate the usefulness of these techniques on nine applications with both fixed and scaled problem sizes. Our results show that, in every case, our method quickly identifies the communication operations that grow to dominate the application's execution time during highly parallel experiments. More importantly, our technique selects operations that a user might not normally locate when using simpler methods.

\subsection{Background}

The analysis of scalability is not a new concept $[6,14]$. Yet many users find scalability analysis of their applications difficult, timeconsuming, and inconclusive. Despite the fact that investigators have proposed numerous metrics, such as speedup, scaled speedup, efficiency, and iso-efficiency, these metrics provide only an abstract and broad view of application scalability behavior. They do not provide specific evidence that allows users to understand and optimize their applications. Worse, the experimental process of measuring application scalability, in practice, can generate an intractable amount of data that can hinder a user's attempts at understanding scalability, because users are simply inundated with lots of uninteresting, redundant data.

Aside from this work, investigators have also proposed scalable visualization techniques for understanding performance data [5, 10, 21]; however, many of these techniques have not been extended to help users understand application scalability. 
Essentially, this previous work has focused on helping users understand the performance data of one application experiment, whereas scalability analysis forces users to integrate performance data from many experiments.

The goal of this work, in contrast, is to develop techniques that promptly and reliably focus a user's attention on the communication operations of their application that limit scalability. With this guidance, a user can easily locate scalability problems and modify their application, if necessary. Our work is empirically driven and directly relevant to existing applications. In fact, our analysis focuses on the Message Passing Interface (MPI) $[9,20]$ because it serves as an important foundation for a large group of high performance applications and communication scalability will become an increasingly important problem as the size of computing systems continues to increase [4, 18, 23].

\subsection{Paper Organization}

The remainder of this paper discusses these issues in more detail. In Section 2, we motivate scalability analysis with a case study and observe that users need automated techniques for scalability analysis. Following this, in Section 3, we introduce our approach to analyzing scalability data with statistical techniques. Then, in Section 0, we evaluate these techniques on numerous MPI applications. Finally, Section 5 concludes the paper with a summary and some interesting research directions.

\section{MOTIVATING EXAMPLE}

To motivate the demands of scalability analysis, we consider a case study of NAS BT [1]. (Section 0 provides complete details of the experimental evaluation.) The goal of this example is to outline the process of manual scalability analysis and to argue for an automated technique.

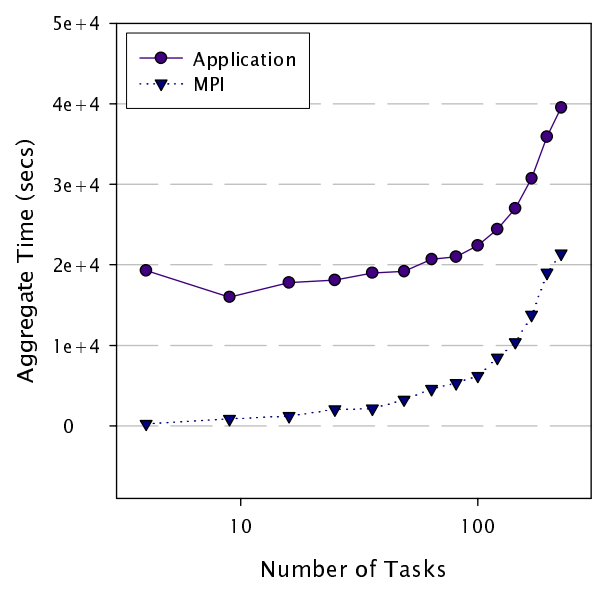

Figure 1: NAS BT Communication Time.

As a first step in analyzing the scalability of BT, we look first at the aggregate runtime of BT from 4 tasks up to 225 tasks as illustrated in Figure 1. More specifically, we divide the time in each task into communication, $T_{\text {comm }}$, and computation, $T_{\text {comp }}$. We capture these values empirically. Using this approach, each task $i$ has a $T_{i, \text { comm }}$, and $T_{i, c o m p}$ that together create $T_{i, a p p}$, the execution time of task $i$. Also, we define $T_{a g g}$ as the processor-time summation across all tasks:

$$
T_{a g g}=\sum_{i=1}^{N} T_{i}=\sum_{i=1}^{N}\left(T_{i, \text { comm }}+T_{i, \text { comp }}\right)
$$

Likewise, we define the aggregate time for communication across all tasks as $T_{\text {agg,comm }}$ :

$$
T_{\text {agg,comm }}=\sum_{i=1}^{N} T_{i, \text { comm }}
$$

Noting that we use the fixed problem size of Class B for this experiment, Figure 1 shows the breakdown of communication time as part of the aggregate application runtime for each experiment size. This analysis quickly illustrates that, for this problem size, BT effectively uses an increasing number of processors up to 81 . However, at this point, BT's communication time drives the application time.

Noticeably, some portion of the communication in BT is increasing; however, this resolution remains too coarse. It is unclear whether all communication operations degrade evenly, or whether one or two communication operations are causing the dramatic increase in communication time.

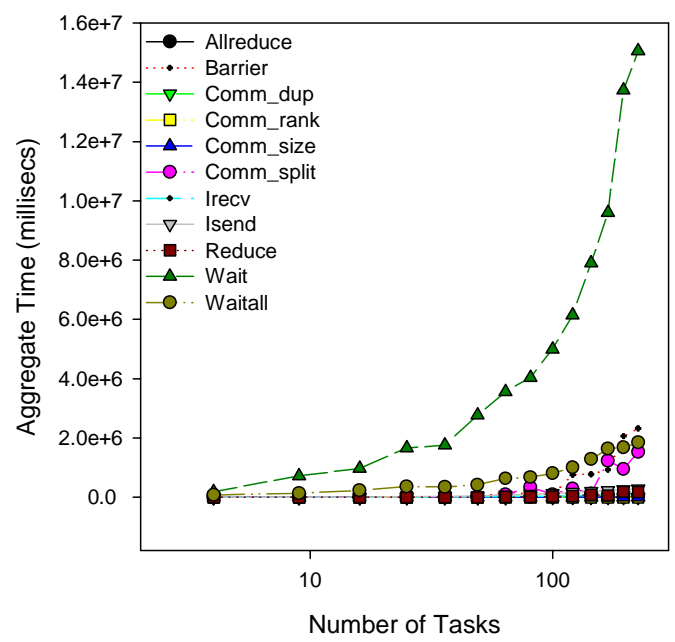

Figure 2: NAS BT Communication Time by Type.

As a next step, we decompose the communication time by operation (e.g., barrier, reduction, send). Many MPI performance analysis tools capture data by the type of MPI call and they do not distinguish among different callsites to the same MPI library routine. Restated, we now have

$$
T_{i, \text { comm }}=\sum_{j}^{\text {allops }} T_{i, j}
$$

where $T_{i, o p}$ provides the total time that task $i$ spent in the op type of communication operation. Figure 2 shows the breakdown of communication operations by type for BT. Viewed in this light, we can focus our scalability analysis on one type of communication operation in BT: Wait, Barrier, Waitall, and Comm_split. Up to 81 tasks, $T_{\text {agg,wait }}$ remains the largest component, but relatively flat; yet beyond 81 tasks, $T_{\text {agg, wait }}$ visibly dominates the communication time. Also, Barrier, Waitall, and Comm_split emerge from the group as the number of tasks increase.

Realistically, however, applications call these operations from multiple callsites. So, as a final step, we split $T_{\text {agg,wait }}$ into 
individual callsites. Recasting $T_{i, \text { wait }}$ as $T_{i \text {,wait,calsite }}$ where

$$
T_{i, \text { wait }}=\sum_{j}^{\text {allcallsites }} T_{i, \text { wait }, j}
$$

we find that not all Wait operations perform similarly as Figure 3 indicates for several select callsites. Indeed, two of the four Waits scale admirably while the other two waits, at $x$ _solve.f:71 and y_solve.f:70, perform much worse.

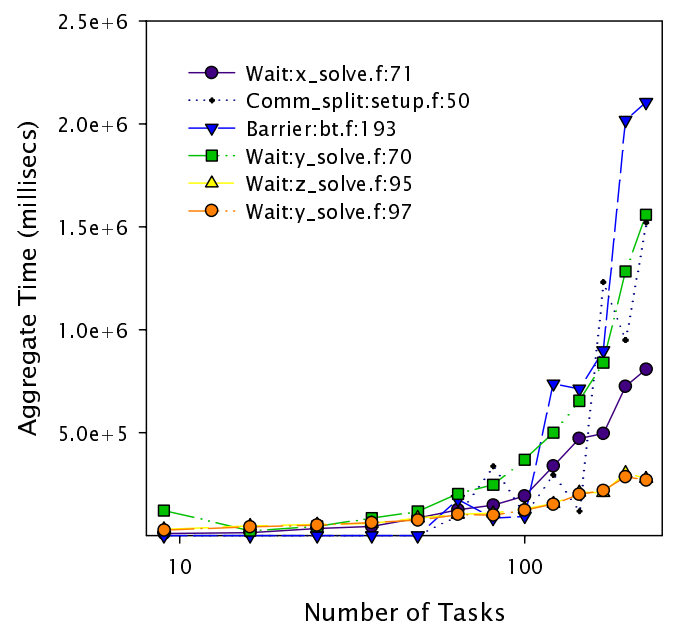

Figure 3: NAS BT communication time for select callsites.

In fact, this decomposition of the communication time by callsite divulges several interesting characteristics about BT. First, many of the data points are quite noisy. Take, for instance, the Comm_split data in Figure 3. These sample points oscillate wildly even though the communication time increases steadily in Figure 1. Our experience indicates that this noise is commonplace, especially on large, production computing systems. Second, the shape of the curves in Figure 3 is strikingly similar to the communication time in Figure 1. All the callsites are relatively flat up to 81 tasks.

In any case, given this information, a user could begin a detailed investigation into why these operations scale poorly, especially when considering that calls to the same communication operation perform distinctively different. The reason for poor scaling could have a number of causes including poor load balance and algorithm design. Also, using this collection of evidence from all callsites, a user could compare calls to the same communication routine and rule out implementation problems, except for the most pathological cases.

\subsection{Observations}

This example illustrates the basic problems encountered by any user when they try to analyze application scalability. First, although the analysis provides some information about scalability, it only provides precise evidence once the user has manually searched through the data to harvest the offensive communication operations. We selected the BT example because it was illustrative; other real world applications will force users to manage similar data for hundreds of MPI callsites across thousands of tasks.

Consider that for one experiment, with $P$ processors executing $n$ communication operations, the analysis can spawn data at the rate of approximately $n \times P$ events at any time $t$. Furthermore, scalability analysis requires data from $E$ experiments, leading to at least $n \times P \times E$ data points. Taken together, these factors can, over a very short period of time, overwhelm most users.

Furthermore, any analysis must account for statistical variations in the experiments as illustrated in our BT example. In this analysis, we ran four experiments at every size of $N$, and then selected the experiment with the smallest running time as the representative for that experiment group. Alternatively, a quantitative analysis should use this additional data to verify and support the conclusions rather than discard it.

\section{STATISTICAL SCALABILITY ANALYSIS}

Given the issues of manual scalability analysis as exposed in Section 2, we propose an alternative solution that relies on statistical analysis $[11,16]$ to extract meaningful relationships from the data. With our solution, users can easily distill the results of their scalability experiments, automatically revealing communication operations that scale poorly.

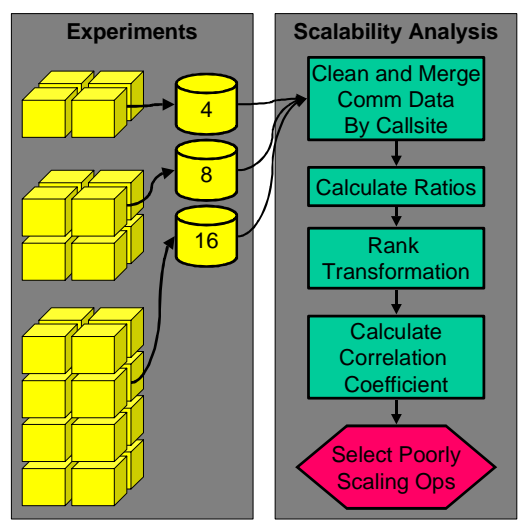

Figure 4: Process for statistical scalability analysis.

Figure 4 illustrates our process for statistical scalability analysis. Our process is composed of two stages. First, the user performance multiple scalability experiments varying the number of tasks, and possibly, the problem size. During the experiment, we record specific timing information about callsites for all communication operations. The user can perform multiple experiments at each configuration; and, in fact, we encourage this.

The next step in our process merges all of the scalability experiment files and then harvests timing information about each callsite. With this information in hand, we calculate the ratio of the aggregate time in each callsite to the aggregate communication time. We, then, rank transform both this ratio and the number of tasks for each experiment. Finally, our process calculates the correlation between the ranked ratio and the ranked number of tasks.

As we will show in Section 4, we find that this non-parametric (or rank) correlation of the ratio of the aggregate time for individual communication operations to aggregate communication time with the number of tasks provides an accurate and stable predictor for identifying communication operations that scale poorly.

\subsection{Performance Data Management}

We organize our data to reflect the stages of scalability analysis presented in Section 2. Since we need callsite information to map 
communication operations to specific locations in the code, our analysis assumes that for all callsites across all tasks in the application, we have the count, cumulative time, and minimum and maximum time. Table 1 shows an example of this performance data from MG.

\begin{tabular}{|l|l|r|r|r|r|r|}
\hline \multicolumn{1}{|c|}{ Op } & Callsite & \multicolumn{1}{c|}{ Task } & Count & Max & Mean & Min \\
\hline Send & mg.f: 1201 & 0 & 487 & 7658 & 963 & 11 \\
\hline Send & mg.f:1201 & 1 & 487 & 6907 & 946 & 12 \\
\hline Send & mg.f: 1201 & 2 & 487 & 5674 & 925 & 11 \\
\hline Send & mg.f:1201 & 3 & 487 & 5707 & 963 & 11 \\
\hline Send & mg.f:1201 & All & 1948 & 7658 & 949 & 11 \\
\hline Allreduce & mg.f:2099 & 0 & 20 & 244 & 132 & 36 \\
\hline Allreduce & mg.f:2099 & 1 & 20 & 484 & 340 & 230 \\
\hline Allreduce & mg.f:2099 & 2 & 20 & 252 & 157 & 65 \\
\hline Allreduce & mg.f:2099 & 3 & 20 & 434 & 355 & 218 \\
\hline Allreduce & mg.f:2099 & All & 80 & 484 & 246 & 36 \\
\hline
\end{tabular}

Table 1: Callsite data for one experiment on NAS MG.

We have constructed a software tool, using the MPI profiling layer [9], to provide straightforward access to this type of data. In addition, we capture the runtime of each task, from start to finish. With this limited information from each experiment, we can do all of the analysis presented in Section 2 and we can easily compute derived values, such as speedup.

For scalability analysis, we have several options for organizing the data. One alternative is to compare callsite values for each task in one experiment to the same callsite in other experiments. This option has the disadvantage that, by definition, experiments have different numbers of tasks, so it does not make sense to compare callsite values task-wise across experiments. As a result, we chose our second strategy for analyzing callsite data: we aggregate the callsite values across all tasks for each experiment and compare the aggregation with similar aggregations for other experiments. Table 2 shows aggregate callsite data for three BT experiments.

\begin{tabular}{|l|r|r|r|}
\hline \multirow{2}{*}{ CALLSITE } & \multicolumn{3}{|c|}{ NUMBER OF TASKS } \\
\cline { 2 - 4 } & \multicolumn{1}{|c|}{9} & \multicolumn{1}{|c|}{16} & \multicolumn{1}{c|}{25} \\
\hline Barrier:bt.f:193 & 11.7 & 26.56 & 58.75 \\
\hline Waitall:copy_faces.f:207 & 137440.8 & 232704 & 358045 \\
\hline Wait:y_solve.f:70 & 122610 & 24240.6 & 44461.2 \\
\hline Comm_split:setup_mpi.f:50 & 23.04 & 39.84 & 81.5 \\
\hline
\end{tabular}

Table 2: Select aggregate callsite data for three BT experiments.

This choice has numerous implications. First, by aggregating the time, we suppress subtle differences across tasks. Second, this aggregation has different results for scaled or fixed problem sizes. As an application scales up with a fixed problem size, the size of messages generally decreases; however, the total number of messages increases. By contrast, as an application scales up with a scaled problem size, the message size remains, generally speaking, constant while the total number of message increases.

\subsection{Correlation Coefficient}

For our data analysis, we turn to the correlation coefficient for two reasons. First, correlation is a relatively simple and wellunderstood technique. It is equally easy to apply many statistical techniques to performance data; however, relating the results of complex statistical measures to source code can be challenging.
Second, other techniques, such as curve fitting, are often difficult to calculate for non-linear functions or noisy sample data, as is regularly the case with performance data.

The sample correlation coefficient, $r$, measures the linear association between two variables and does not depend on the units of measurements. The standard equation for the linear correlation coefficient is

$$
r=\frac{\sum_{i}\left(x_{i}-\bar{x}\right)\left(y_{i}-\bar{y}\right)}{\sqrt{\sum_{i}\left(x_{i}-\bar{x}\right)^{2}} \sqrt{\sum_{i}\left(y_{i}-\bar{y}\right)^{2}}} .
$$

The value of $r$ must be between -1 and +1 . Quantitatively, $r$ measures the strength of the linear association between two variables. If $r$ is near 0 , it implies no association between the variables, or that they are uncorrelated. Otherwise, the sign of $r$ specifies the direction of the association. If $r$ is negative, it implies an inclination for one value in the pair to be larger than its average when the other is smaller than its average. If $r$ is positive, it suggests a tendency for one value of the pair to be large when the other value is large and also for both values to be small together.

Disappointingly, however, the linear correlation coefficient suffers several limitations. First, it assumes that the underlying distributions are normal. Second, nonlinear associations can exist that are not revealed by the linear correlation coefficient. Third, linear correlation can be very sensitive to outliers, showing an association between variables when it does not exist. Finally, correlation does not imply causality in the association. We address these limitations by using rank transformation and by applying the correlation computation in a very restricted way.

In addition to rank transformation, we can argue that, in our context, our data does not have nonlinear relationships and that it does imply causality. Our argument relies on the fact that, as we have designed it, the application's total communication time is the sum of all the application's communications operations. An increase in any operation's time directly increases the total communication time. So, it is a linear and casual relationship by design.

Another important point is that correlation can accept any number of scalability experiments, including experiments with identical configurations. This only improves the analysis either by strengthening or weakening the relationship between individual communication operations and the total communication time. That is, if two identically configured experiments have the same communication times yet produce drastically different measurements for one callsite, then any conclusion about that callsite must be interpreted carefully.

\subsection{Rank Transformation}

Because we are concerned about the normality assumption of the linear correlation technique and the effect of outliers in our measurements, we perform non-parametric or rank correlation $[11,16]$ on our data. Rank transformation is the process of replacing the actual observations by their ranks. Assume that we are given $\mathrm{N}$ pairs of measurements: $X, Y$. The key notion of nonparametric correlation is that by replacing the value of each $x$ by the value of its rank among all other $x$ s in the sample. The transformed list will be drawn from a uniform distribution function from the integers between 1 and $\mathrm{N}$, inclusive. The values for $Y$ are transformed in the same way. When some of the values are identical, we assign these values the midrank for their range: 
the mean of the ranks of that these values would have occupied if their values were different. In particular, we use the Spearman rank correlation coefficient:

$$
r_{s}=\frac{\sum_{i}\left(R_{i}-\bar{R}\right)\left(S_{i}-\bar{S}\right)}{\sqrt{\sum_{i}\left(R_{i}-\bar{R}\right)^{2}} \sqrt{\sum_{i}\left(S_{i}-\bar{S}\right)^{2}}} .
$$

We prefer the rank transformed correlation because it is less likely to be distorted by non-normality and unusual observations.

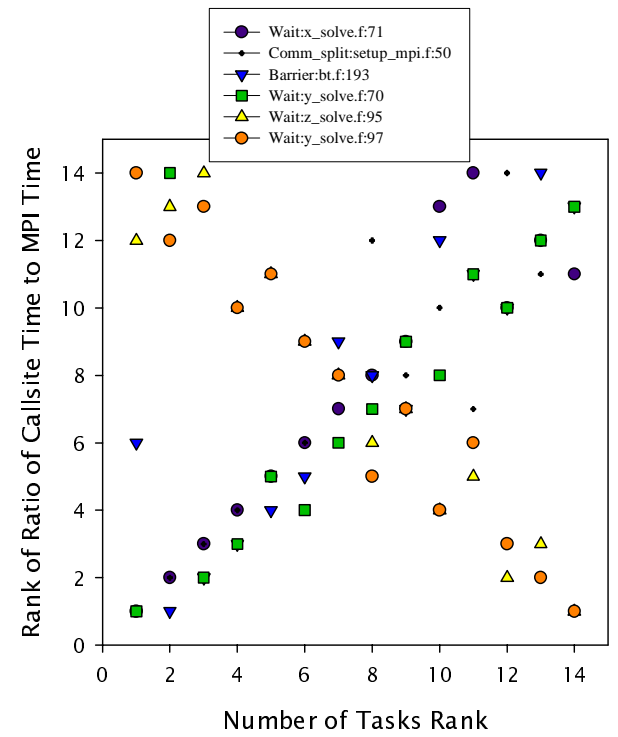

Figure 5: Example rank scatterplot of BT callsites.

Figure 5 illustrates a scatterplot of several BT callsites after the ranking. Associations clearly emerge between the number of tasks and several callsites in this figure. The first four callsites, Wait:x_solve.f:71, Comm_split:setup_mpi.f:50, Barrier:bt.f:193, and Wait:y_solve.f:70, are positively correlated with the number of tasks, while Wait:z_solve.f:95 and Wait:y_solve.f:97 are negatively correlated.

\section{EXPERIMENTAL EVALUATION}

To explore our hypothesis of using rank correlation to analyze scalability, we have constructed an operational prototype for MPI applications. With this prototype, we empirically evaluated nine applications, extracted from the NAS Parallel and the ASCI Compact Benchmarks.

\subsection{Experiment Overview}

To capture information about the communication operations within these MPI applications, we extract a limited set of information about each MPI call during application execution, as discussed in Section 3. Unlike trace-based performance analysis tools [8, 17, 19], our lightweight profiler captures only fundamental performance data about MPI callsites: number of times called and cumulative time spent in the call. In fact, Table 1 shows profiler output for one NAS MG experiment. Callsite is location of the MPI function in the user's the source code. Rank identifies each MPI task's rank within communicator MPI_COMM_WORLD. All represents the aggregate values across all tasks for that callsite. Count provides the number of times that function at that callsite was called. Max, Min, and Mean capture the maximum, minimum, and mean duration, respectively, for each callsite.

We have found that this profiling technique balances the requirements of low overhead and manageable data volume with the need for overview information about an application's communication. Furthermore, the amount of data generated with this lightweight technique is invariant to the length of the application's run time. Put another way, the amount of profiler output for an application experiment is effectively constant whether the application runs for 5 minutes or 5 days. Still, tracing can provide a wealth of information about application communication performance; its chronological description of application events is more general than techniques such as our profiling. Note, however, that users can apply the techniques developed here to information gathered in trace files.

For these evaluations, we analyze each application from start to finish; therefore, these results contain initialization and setup phases that might not be included in strict algorithmic evaluations.

\subsection{Platform}

We ran our tests in the batch partition of the ASCI Blue Pacific combined technology refresh (CTR) SP at Lawrence Livermore National Laboratory. This machine is composed of $332 \mathrm{Mhz} 604 \mathrm{e}$ 4-way SMP nodes, totaling 1344 CPUs. Each compute node has a peak performance of 2.656 GigaOPS. The $604 \mathrm{e}$ processor has one floating-point unit and one load/store unit. Its $32 \mathrm{~KB} \mathrm{L1}$ cache is 4 way associative with 32 byte cache lines and L1 uses an LRU replacement scheme. The processor has a 500KB L2 cache. At the time of our tests, the batch partition had 305 nodes and the operating system was AIX 4.3.3. We compiled the various tests with the IBM XL compilers and used IBM's MPI library in userspace mode. Each SMP node contains 1.5 GB main memory for a total of 504 GB system memory. Node to node bi-directional bandwidth is $150 \mathrm{Mbyte} / \mathrm{s}$. Our test jobs ran on dedicated nodes, although other jobs were concurrently using the network.

\subsection{Application Results}

We tested six of the benchmarks from NAS Parallel Benchmark 2.3 suite [1]: BT, SP, MG, FT, LU, and CG. These NAS benchmarks are fixed-problem size; our experiments use class B problems. We also tested other applications from the ASCI Compact Benchmark suite: sPPM, Sweep3d, and SMG2000. These benchmarks scale problem sizes with the number of tasks. Table 4 outlines the experiment configurations for these applications. We performed the experiment at least three times at each configuration.

Table 3 presents the rank correlation results for all the applications we studied. We limited the callsites included in the table to those callsites that exceeded a threshold of $1 \%$ for at least one of the experiments on that application.

For clarity, we graph only the data points for each experiment configuration whose aggregate execution time was the minimum for that configuration.

\subsubsection{NAS Parallel Benchmarks}

Figure 6 shows our scaling results for NAS benchmarks. All of the NAS benchmarks scale well, given that they are constrained by their fixed problem size. As other researchers have noted [24], these benchmarks effectively utilize an increasing number of processors up to a limit where they suffer from their inevitable 
growth in communication overhead and from their shrinking local problem size.

The benchmark applications NAS SP and BT represent computational fluid dynamics (CFD) applications that solve systems of equations resulting from an approximately factored implicit finite-difference discretization of the Navier-Stokes

\begin{tabular}{|l|c|}
\hline \multicolumn{1}{|c|}{ bt.B } & $\mathbf{r}_{\mathbf{s}}$ \\
\hline Wait:x_solve.f:71 & 0.93 \\
\hline Comm_split:setup_mpi.f:50 & 0.90 \\
\hline Barrier:bt.f:193 & 0.89 \\
\hline Wait:y_solve.f:70 & 0.64 \\
\hline Wait:y_solve.f:0 & 0.44 \\
\hline Wait:z_solve.f:68 & 0.42 \\
\hline Wait:x_solve.f:0 & -0.06 \\
\hline Wait:x_solve.f:99 & -0.07 \\
\hline Wait:y_solve.f:69 & -0.32 \\
\hline Wait:z_solve.f:0 & -0.32 \\
\hline Barrier:bt.f:147 & -0.44 \\
\hline Wait:x_solve.f:70 & -0.47 \\
\hline Wait:y_solve.f:98 & -0.53 \\
\hline Wait:z_solve.f:96 & -0.56 \\
\hline Wait:z_solve.f:67 & -0.86 \\
\hline Wait:x_solve.f:98 & -0.92 \\
\hline Waitall:copy_faces.f:207 & -0.95 \\
\hline Wait:z_solve.f:95 & -0.96 \\
\hline Wait:y_solve.f:97 & -0.97 \\
\hline
\end{tabular}

\begin{tabular}{|l|c|}
\hline \multicolumn{1}{|c|}{ sp.B } & $\mathbf{r}_{\mathbf{s}}$ \\
\hline Comm_split:setup_mpi.f:51 & 0.85 \\
\hline Barrier:sp.f:189 & 0.77 \\
\hline Waitall:y_solve.f:74 & 0.71 \\
\hline Bcast:sp.f:102 & 0.65 \\
\hline Allreduce:error.f:50 & 0.35 \\
\hline Isend:x_solve.f:335 & 0.18 \\
\hline Waitall:x_solve.f:81 & -0.03 \\
\hline Barrier:sp.f:143 & -0.19 \\
\hline Waitall:z_solve.f:73 & -0.39 \\
\hline Waitall:y_solve.f:380 & -0.78 \\
\hline Waitall:z_solve.f:377 & -0.91 \\
\hline Waitall:x_solve.f:388 & -0.92 \\
\hline Waitall:copy_faces.f:208 & -0.94 \\
\hline
\end{tabular}

\begin{tabular}{|l|r|}
\hline \multicolumn{1}{|c|}{ ft.B } & $\mathbf{r}_{\mathbf{s}}$ \\
\hline Bcast:ft.f:443 & 0.87 \\
\hline Comm_split:ft.f:506 & 0.86 \\
\hline Barrier:ft.f:118 & -0.41 \\
\hline Alltoall:ft.f:1283 & -0.91 \\
\hline Reduce:ft.f:1662 & -0.94 \\
\hline
\end{tabular}

\begin{tabular}{|l|c|}
\hline \multicolumn{1}{|c|}{ sweep3d } & $\mathbf{r}_{\mathbf{s}}$ \\
\hline Barrier:mpi_stuff.f:337 & 0.50 \\
\hline Bcast:mpi_stuff.f:248 & 0.09 \\
\hline Send:mpi stuff.f:128 & -0.10 \\
\hline Recv:mpi_stuff.f:155 & -0.33 \\
\hline Allreduce:mpi_stuff.f:270 & -0.36 \\
\hline Allreduce:mpi_stuff.f:316 & -0.73 \\
\hline Comm_size:mpi_stuff.f:36 & -0.86 \\
\hline
\end{tabular}

equations. The SP and BT algorithms have a similar structure; each solves three sets of uncoupled systems of equations. BT solves block-tridiagonal systems of $5 \times 5$ blocks; SP solves scalar pentadiagonal systems resulting from full diagonalization of the approximately factored scheme.

\begin{tabular}{|l|c|}
\hline \multicolumn{1}{|c|}{ mg.B } & $\mathbf{r}_{\mathbf{s}}$ \\
\hline Wait:mg.f:1315 & 0.89 \\
\hline Wait:mg.f:1542 & 0.70 \\
\hline Barrier:mg.f:99 & 0.67 \\
\hline Barrier:mg.f:2089 & 0.62 \\
\hline Allreduce:mg.f:1004 & 0.48 \\
\hline Allreduce:mg.f:2139 & 0.43 \\
\hline Allreduce:mg.f:1001 & 0.41 \\
\hline Allreduce:mg.f:2099 & 0.41 \\
\hline Allreduce:mg.f:2115 & 0.39 \\
\hline Allreduce:mg.f:2123 & 0.33 \\
\hline Barrier:mg.f:233 & 0.09 \\
\hline Reduce:mg.f:256 & 0.01 \\
\hline Bcast:mg.f:133 & -0.06 \\
\hline Send:mg.f:1215 & -0.11 \\
\hline Irecv:mg.f:1160 & -0.13 \\
\hline Send:mg.f:1247 & -0.15 \\
\hline Send:mg.f:1233 & -0.27 \\
\hline Send:mg.f:1265 & -0.33 \\
\hline Comm rank:mg.f:86 & -0.36 \\
\hline Send:mg.f:1201 & -0.41 \\
\hline Send:mg.f:1279 & -0.48 \\
\hline
\end{tabular}

\begin{tabular}{|l|l|}
\hline \multicolumn{1}{|c|}{ sppm } & $\mathbf{r}_{\mathrm{s}}$ \\
\hline Allreduce:main.f:1392 & 0.81 \\
\hline Wait:bdrys.f:1247 & 0.75 \\
\hline Wait:bdrys.f:1248 & 0.73 \\
\hline Wait:bdrys.f:928 & 0.72 \\
\hline Wait:bdrys.f:3596 & 0.63 \\
\hline Wait:bdrys.f:2421 & 0.54 \\
\hline Allreduce:main.f:1025 & 0.48 \\
\hline Wait:bdrys.f:2422 & 0.46 \\
\hline Wait:bdrys.f:2953 & 0.45 \\
\hline Wait:bdrys.f:2954 & 0.34 \\
\hline Wait:bdrys.f:2102 & -0.03 \\
\hline Wait:bdrys.f:1780 & -0.24 \\
\hline Wait:bdrys.f:3595 & -0.24 \\
\hline Wait:bdrys.f:927 & -0.28 \\
\hline Wait:bdrys.f:1779 & -0.33 \\
\hline Wait:bdrys.f:3276 & -0.50 \\
\hline Wait:bdrys.f:3275 & -0.82 \\
\hline Wait:bdrys.f:2477 & -0.84 \\
\hline Wait:bdrys.f:606 & -0.85 \\
\hline Wait:bdrys.f:3651 & -0.88 \\
\hline Wait:bdrys.f:605 & -0.88 \\
\hline Wait:bdrys.f:2101 & -0.94 \\
\hline
\end{tabular}

\begin{tabular}{|l|r|}
\hline \multicolumn{1}{|c|}{ Iu.B } & \multicolumn{1}{|c|}{$\mathbf{r}_{\mathbf{s}}$} \\
\hline Allreduce:I2norm.f:55 & 0.75 \\
\hline Bcast:bcast_inputs.f:28 & 0.73 \\
\hline Send:exchange_3.f:275 & 0.46 \\
\hline Recv:exchange_1.f:30 & 0.45 \\
\hline Send:exchange_3.f:209 & 0.41 \\
\hline Send:exchange_1.f:149 & 0.30 \\
\hline Send:exchange_1.f:130 & -0.01 \\
\hline Recv:exchange_1.f:48 & -0.04 \\
\hline Send:exchange_1.f:166 & -0.08 \\
\hline Send:exchange_1.f:113 & -0.13 \\
\hline Recv:exchange_1.f:86 & -0.13 \\
\hline Send:exchange_3.f:139 & -0.18 \\
\hline Recv:exchange_1.f:68 & -0.21 \\
\hline Send:exchange_3.f:73 & -0.25 \\
\hline Wait:exchange_3.f:288 & -0.44 \\
\hline Wait:exchange_3.f:222 & -0.46 \\
\hline Wait:exchange_3.f:152 & -0.83 \\
\hline Wait:exchange_3.f:86 & -0.92 \\
\hline
\end{tabular}

\begin{tabular}{|l|r|}
\hline \multicolumn{1}{|c|}{ cg.B } & \multicolumn{1}{|c|}{$\mathbf{r}_{\mathbf{s}}$} \\
\hline Barrier:cg.f:411 & 0.91 \\
\hline Wait:cg.f:1221 & 0.90 \\
\hline Wait:cg.f:1275 & 0.90 \\
\hline Wait:cg.f:1397 & 0.85 \\
\hline Wait:cg.f:1059 & 0.58 \\
\hline Wait:cg.f:1150 & 0.44 \\
\hline Irecv:cg.f:1318 & 0.43 \\
\hline Wait:cg.f:1177 & -0.23 \\
\hline Send:cg.f:1326 & -0.66 \\
\hline Send:cg.f:1143 & -0.76 \\
\hline Send:cg.f:1354 & -0.76 \\
\hline Send:cg.f:1170 & -0.79 \\
\hline
\end{tabular}

\begin{tabular}{|l|r|}
\hline \multicolumn{1}{|c|}{ smg2000 } & \multicolumn{1}{c|}{$\mathbf{r}_{\mathbf{s}}$} \\
\hline Allreduce:timing.c:419 & 0.93 \\
\hline Allreduce:struinprod.c:107 & 0.92 \\
\hline Waitall:coarsen.c:542 & 0.83 \\
\hline Waitall:coarsen.c:491 & 0.80 \\
\hline Barrier:smg2000.c:227 & 0.71 \\
\hline Allgather:struct_grid.c:366 & 0.62 \\
\hline Waitall:comm.c:667 & -0.27 \\
\hline Irecv:comm.c:485 & -0.98 \\
\hline Isend:comm.c:492 & -0.99 \\
\hline Type_commit:comm.c:1487 & -0.99 \\
\hline Type_free:comm.c:1405 & -0.99 \\
\hline Type_free:comm.c:1413 & -0.99 \\
\hline
\end{tabular}

Table 3: Rank Correlation Results. 


\begin{tabular}{|l|l|c|}
\hline Application & \multicolumn{1}{|c|}{$\begin{array}{c}\text { Scaled problem, } \\
\text { Problem size }\end{array}$} & $\begin{array}{c}\text { Experiment } \\
\text { range }\end{array}$ \\
\hline NAS BT & No, Class B & $4-225$ \\
\hline NAS CG & No, Class B & $2-256$ \\
\hline NAS FT & No, Class B & $8-256$ \\
\hline NAS LU & No, Class B & $4-256$ \\
\hline NAS MG & No, Class B & $2-256$ \\
\hline NAS SP & No, Class B & $4-225$ \\
\hline $\begin{array}{l}\text { SMG2000 } \\
\text { [0] }\end{array}$ & Yes, 10x10x10 per task & $2-1024$ \\
\hline sPPM & Yes, $128^{3}$ per task & $2-768$ \\
\hline Sweep3D & Yes, 6x6x300 per task & $2-512$ \\
\hline
\end{tabular}

Table 4: Applications.

The analysis of our motivating example, BT, exposes that a Wait, a Comm_split, and a Barrier grow considerably with the number of tasks. The strongest correlation is Wait:x_solve.f:71. Referring back to Figure 3, we can see that this Wait is consistently increasing as the number of tasks increases. Upon closer examination, this Wait completes the first Irecv of the solver for the $\mathrm{X}$-direction. This is the first message passing operation in the sequence, so any load-imbalance up to that point is credited toward this Wait. The other two operations, Comm_split and Barrier, increase as the number of tasks increase as well. Note that although they do consume more time than the Wait, they are much less consistent. Hence, they have a lower rank correlation.

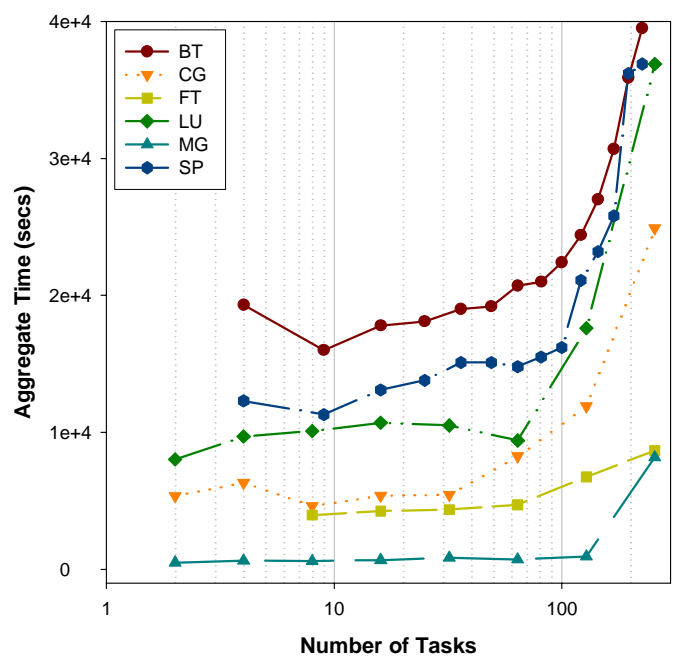

Figure 6: NAS NPB Class B Scaling.

Interestingly, the rank correlation for SP also positions a Comm_split, a Barrier, and a Waitall as the most closely correlated with the number of tasks. It structure is very similar to BT.

NAS CG computes an approximation to the smallest eigenvalue of a large, sparse, symmetric positive definite matrix, which is characteristic of unstructured grid computations.

Our analysis of CG rates a Barrier and several Wait operations as the main culprits that scale poorly. As Table 3 and Figure 7 show, the remaining Wait operations have a strong positive correlation with the number of tasks. On the other hand, the Barrier call is used for initialization and it increases from about 1 millisecond for 2 tasks up to 80 seconds for one experiment at 256 tasks (not shown).

NAS FT, which solves a 3-D partial differential equation using
FFTs, has few MPI calls, relying entirely on collective operations. FT relies mainly on one Alltoall for periodic exchanges of data among tasks for the FFT. As the number of tasks grows, though, two relatively obscure collective operations dominate communication time: a Bcast and a Comm_split. This example demonstrates one of the most important features of our analysis technique. The Alltoall operation is a very important operation for the FFT as shown in Figure 8; however, its contribution to the communication time remains relatively constant when scaling from 2 to 256 tasks. Instead, our rank correlation technique clearly identifies the two other collectives, Bcast and Comm_split, as the callsites that increase with the number of tasks.

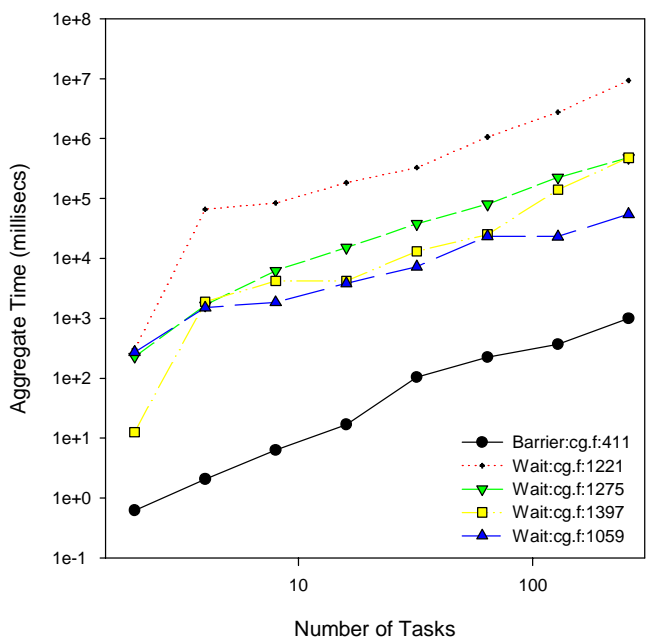

Figure 7: Aggregate time for select CG callsites.

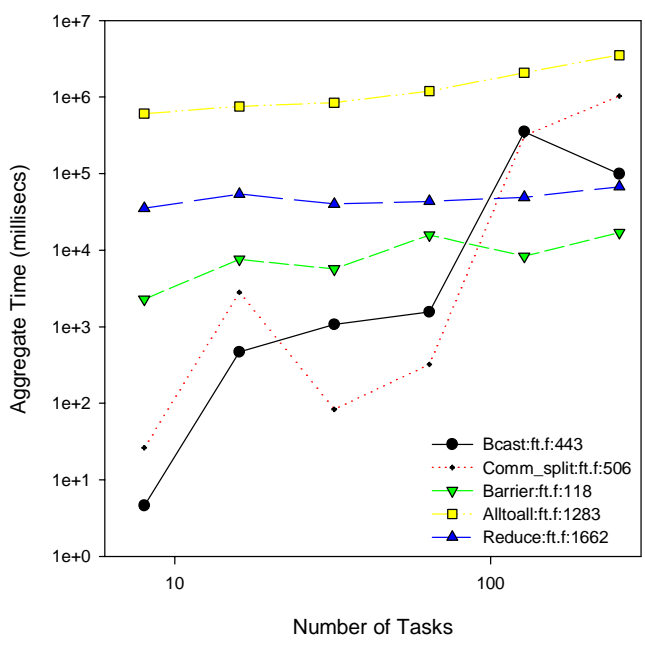

Figure 8: Aggregate time for FT callsites.

NAS LU solves a regular-sparse, block 5x5 lower and upper triangular system using a symmetric successive over-relaxation (SSOR) numerical scheme.

Again, collective operations dominate the top two spots for our evaluation for LU: an Allreduce and a Bcast. More importantly, only 6 of 18 listed communication operations have a positive correlation to the number of tasks. Figure 9 helps make this result 
understandable. Once more, we see these two collectives have a very small portion of the communication time at 4 tasks. They, then, grow consistently to hold a large portion of the communication time at 256 tasks. Contrast this result with Wait:exchange_3.f:86 and the result is more conspicuous. For this particular Wait, the aggregate time remains almost constant during our scaling experiments, even though, it holds a large portion of the communication time.

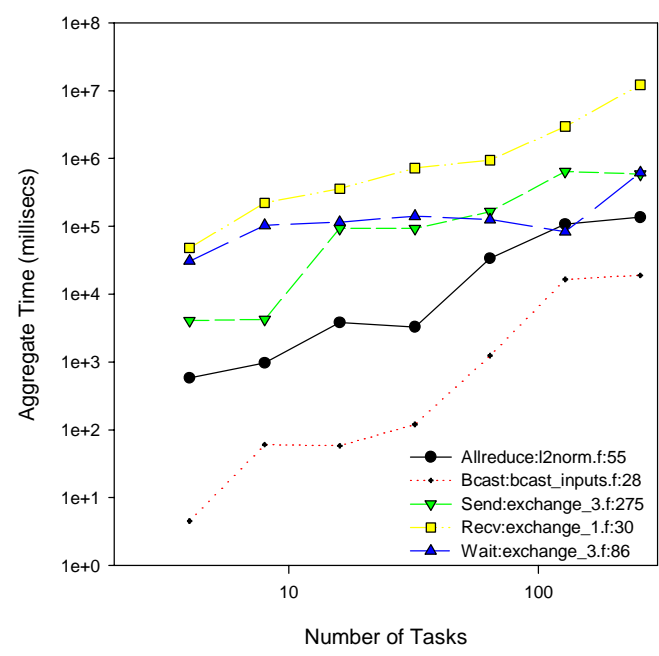

Figure 9: Aggregate time for select $\mathrm{LU}$ callsites

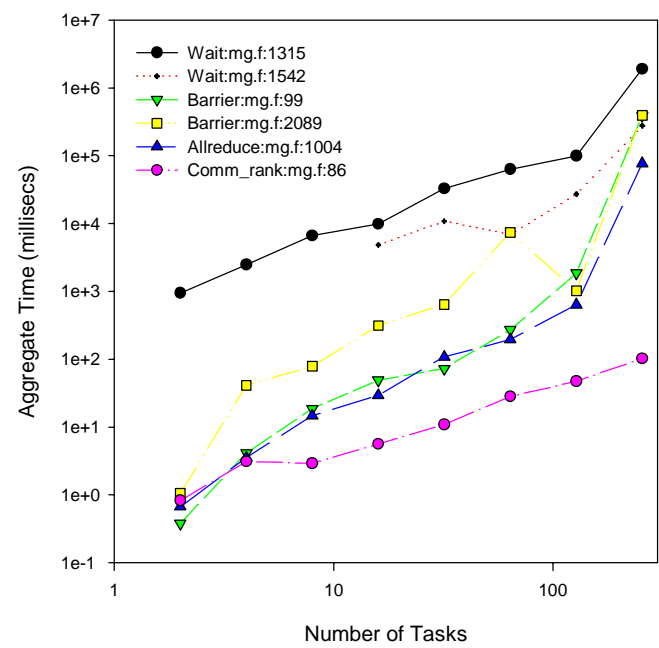

Figure 10: Aggregate time for select MG callsites

NAS MG is a kernel benchmark that runs a simplified multigrid solver to compute a three-dimensional potential field. MG has only two positively correlated communication operations that are not collectives: two Waits. The remaining operations include three Barriers and six Allreduces.

As Figure 10 demonstrates, Wait:mg.f:1315 consumes most of the communication time and it grows steadily with the number of tasks. Wait:mg.f:1542 also holds a top position and maintains that position as the number of tasks grows. This callsite begins at 16 tasks because the problem decomposition at smaller task sizes did not explore all the control flow paths. Compare these positively correlated callsites with Comm_rank:mg.f:86, which also increases as does the number of tasks. Comm_rank:mg.f:86 is negatively correlated at -0.36 with the nubmer of tasks because as the communication time increases dramatically from 128 to 256 tasks (from Figure 6), the aggregate time for this callsite does not change noticeably and its portion of the communication time shrinks.

\subsubsection{ASCI Benchmarks}

Figure 11 illustrates the results of our scaling experiments for the three ASCI Benchmarks. All three of these benchmarks have scaled problem sizes, so we were able to run much larger problems and in the case of SMG, we ran up to 1024 tasks.

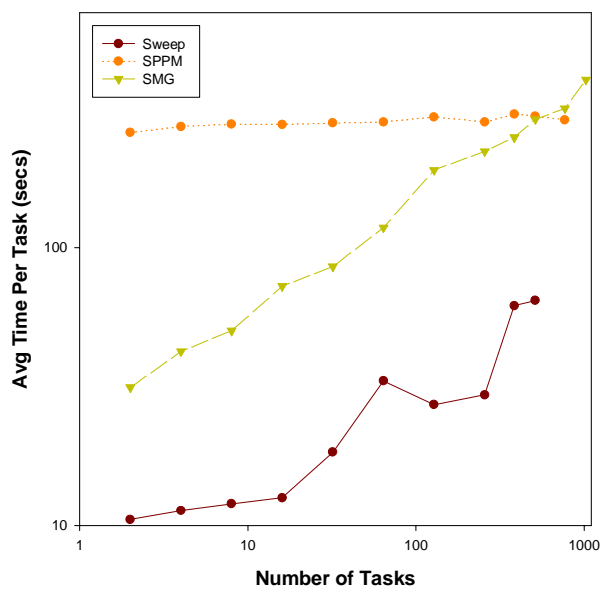

Figure 11: ASCI Benchmark Scaling.

sPPM [15] solves a 3D gas dynamics problem on a uniform Cartesian mesh, using a simplified version of the Piecewise Parabolic Method. The algorithm makes use of a split scheme of $\mathrm{X}, \mathrm{Y}$, and Z Lagrangian and remap steps, which are computed as three separate sweeps through the mesh per timestep. Message passing provides updates to ghost cells from neighboring domains three times per timestep. The sPPM code is written in Fortran 77 with some $\mathrm{C}$ routines. The code uses the asynchronous message operations: MPI_Irecv and MPI_Isend.

Our analysis of SPPM confirmed our earlier investigation [22] and reinforced the experience of others [15]: SPPM scales very well. As illustrated in Table 3, our rank correlation identifies Allreduce:main.f:1392 main culprit while a host of Waits dominate the other positively correlated operations. As Figure 12 illustrates, Allreduce:main.f:1392 rockets from a very small portion of the communication time at 2 tasks up to practically dominating the communication time at 768 tasks. Closer scrutiny of the code reveals that the two Allreduces are called in sequence with the first one, Allreduce:main.f:1392, bearing the brunt of any load imbalance. SPPM calls the second Allreduce at Allreduce:main.f:1025 immediately after the first one.

SMG2000 [2] is a parallel semicoarsening multigrid solver for the linear systems arising from finite difference, finite volume, or finite element discretizations of the diffusion equation, $\nabla \cdot(D \nabla u)+\sigma u=f$ on logically rectangular grids. The code solves both $2 \mathrm{D}$ and $3 \mathrm{D}$ problems with discretization stencils of up to 9 -point in $2 \mathrm{D}$ and up to 27 -point in $3 \mathrm{D}$. This code is a 
distributed memory application based on C and MPI.

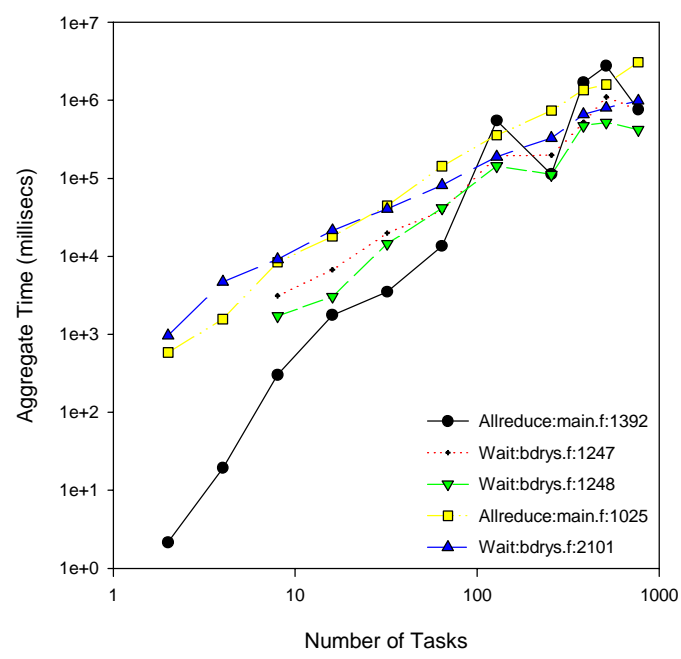

Figure 12: Select callsites for SPPM.

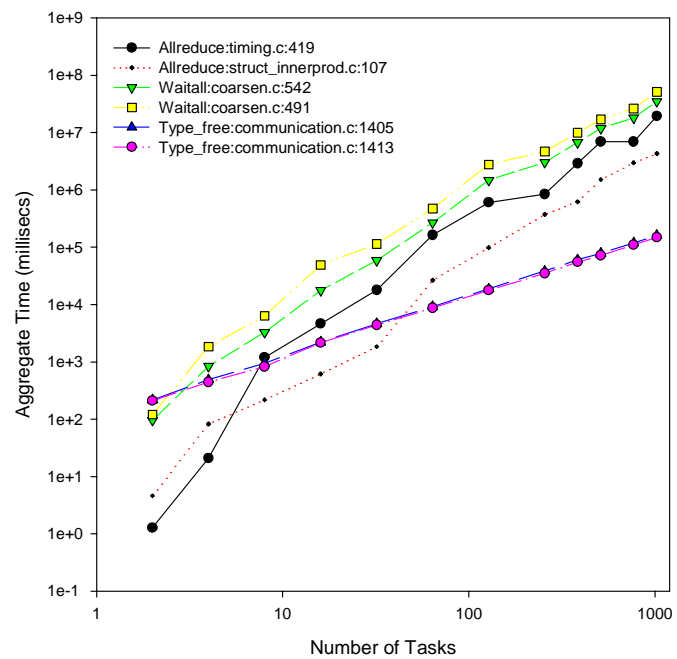

Figure 13: Select callsites for SMG2000.

SMG2000 scaling suffers from several collective operations. Four of the six operations are collectives with two Waitalls also having a strong positive correlation. Surprisingly, the SMG2000 rank correlation shows a large gap between positive and negative correlated operations. Six communication operations are strongly correlated to the number of tasks, where the lowest positive coefficient is 0.62 . Figure 13 clarifies this difference. As before, these collective operations consume a very small portion of the communication time at 2 tasks and then they rise to the top positions at 1024 tasks. Once again, rank correlation has helped to identify the callsites that grow to consume more communication time as the number of tasks group.

Sweep3D [12] is a solver for the 3-D, time-independent, particle transport equation on an orthogonal mesh. The solver computes along wavefronts in the mesh in eight diagonal directions through the cube. This code is built on FORTRAN and MPI.

Sweep3D scaling mirrors our earlier analysis where two collectives emerge to dominate practically the communication time. Unfortunately, Sweep3D wraps MPI communication routines in its own communication abstraction procedures, so all of the communication performance data is mapped into seven operations. Effectively, our data needs one more level of from the stack, so that it can discriminate among calls to these user-defined abstractions. We are currently implementing this feature.

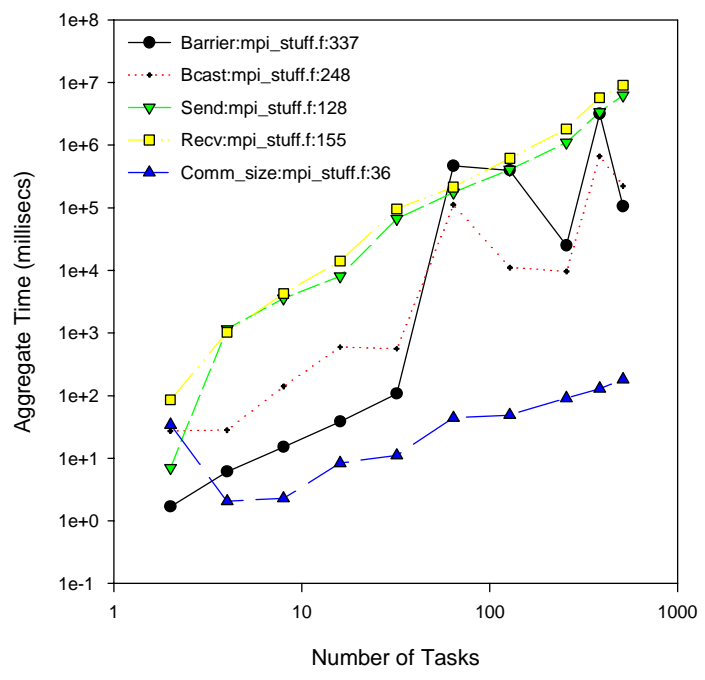

Figure 14: Select callsites for Sweep3D.

\subsection{Observations}

The evaluation of our proposed technique for scalability analysis on nine applications produces several interesting observations. First, rank correlation of the number of tasks with the ratio of callsite times to the overall communication time clearly identifies trends in the communication operations of the applications we evaluated. In every case, this automated technique highlighted the communication operations that grow to dominate communication time as the number of tasks increase.

Second, this type of correlation identifies callsites that simpler methods of analysis would not. Simply put, our analysis extracts trends from the data that could be easily overlooked by just looking at one experiment at a large task count. We can apply rank correlation to a large set of experiments and allow it to grade callsites based on all the evidence it is presented.

Third, rank correlation is necessary in our example; however, the rank transformation discards some useful information including the magnitude of the variance. For instance, this conversion makes two sets of monotonically-increasing data samples exactly the same even though one set might change by a factor of 100 more than the other set. Still, the rank transformation eliminates more concerns than it introduces. It safely guarantees a normal distribution and it controls outliers.

Finally, it is important to note that both positive and negative correlation provides useful information. For instance, in the case of NAS FT, these correlations unmistakably separated the collective operations into two groups.

On reflection, our technique could benefit from several improvements as well. In particular, our analysis of Sweep3D was thwarted by the aliasing of performance data into the user-defined communication wrappers. 
Our experiences also indicate that although this analysis uses only timing data, it can serve as a first-order approximation at scalability and other performance problems. With this information a user can easily interrogate the original data, or perform a trace of the offensive operations for more detailed performance data.

\section{CONCLUSIONS}

In this paper, we have proposed and evaluated a novel technique for identifying communication operations that scale poorly. Our technique uses non-parametric (or rank) correlation of the number of tasks to the ratio of the time for individual communication operations to the overall communication time. Our initial results with these applications on up to 1024 processors show that our correlation technique automatically and correctly identifies the poorly scaling operations in every case. We have also successfully used this technique to analysis applications with 1536 processors on the ASCI White initial delivery system and with over two hundred individual MPI callsites. More importantly, our technique identifies performance problems in a way that a user can easily relate to the design of their application. Our experiments showed that our technique extracts trends from the performance data that are very important, but not obvious. Our experiments also show that similar communication operations do not scale similarly.

We believe that this technique is applicable to other types of performance analysis. We are beginning to explore, for example, the use of this technique on data analysis for hardware performance counters across hundreds or thousands of concurrent application regions. Accordingly, we are also investigating automated techniques that robustly discover other phenomena including load-balancing problems.

\section{ACKNOWLEDGEMENTS}

This work was performed under the auspices of the U.S. Dept. of Energy by University of California LLNL under contract W-7405Eng-48. LLNL Document Number UCRL-JC-142611.

\section{REFERENCES}

[1] D. Bailey, E. Barszcz et al., "The Nas Parallel Benchmarks (94)," NASA Ames Research Center, RNR Technical Report RNR-94-007, 1994.

[2] P.N. Brown, R.D. Falgout et al., "Semicoarsening Multigrid on Distributed Memory Machines," SIAM Journal on Scientific Computing, 21(5):1823-34, 2000.

[3] A.C. Calder, B.C. Curtis et al., "High-Performance Reactive Fluid Flow Simulations Using Adaptive Mesh Refinement on Thousands of Processors," Proc. SC2000: High Performance Networking and Computing Conf. (electronic publication), 2000.

[4] M. Calzarossa, L. Massari et al., "Parallel Performance Evaluation: The Medea Tool," Proc. High-Performance Computing and Networking (HPCN Europe 1996), 1996, pp. 522-9.

[5] S.K. Card, B. Shneiderman et al., Readings in Information Visualization: Using Vision to Think. San Francisco, CA: Morgan Kaufmann Publishers, 1999.

[6] D.E. Culler, J.P. Singh et al., Parallel Computer Architecture: A Hardware Software Approach. San Francisco: Morgan Kaufmann Publishers, 1999.

[7] I. Foster, Designing and Building Parallel Programs: Concepts and Tools for Parallel Software Engineering. Reading, MA: Addison-Wesley, 1995.
[8] G.A. Geist, M.T. Heath et al., "A Users' Guide to Picl - a Portable Instrumented Communication Library," Oak Ridge National Laboratory, P.O.Box 2009, Bldg. 9207-A, Oak Ridge, TN 37831-8083 1991.

[9] W. Gropp, E. Lusk et al., Using Mpi: Portable Parallel Programming with the Message-Passing Interface, 2nd ed. Cambridge, MA: MIT Press, 1999.

[10] M.T. Heath, A.D. Malony et al., "Parallel Performance Visualization: From Practice to Theory," IEEE Parallel \& Distributed Technology: Systems \& Applications, 3(4):4460, 1995.

[11] R.A. Johnson and D.W. Wichern, Applied Multivariate Statistical Analysis, 4 ed. Englewood Cliffs, New Jersey, USA: Prentice-Hall, 1998.

[12] K.R. Koch, R.S. Baker et al., "Solution of the First-Order Form of the 3-D Discrete Ordinates Equation on a Massively Parallel Processor," Trans. Amer. Nuc. Soc., 65(198), 1992.

[13] V. Kumar, A. Grama et al., Introduction to Parallel Computing: Design and Analysis of Algorithms. Redwood City, Calif.: Benjamin/Cummings Pub. Co., 1994.

[14] V. Kumar and A. Gupta, "Analyzing Scalability of Parallel Algorithms and Architectures," Journal of Parallel and Distributed Computing, 22(3):379-91, 1994.

[15] A.A. Mirin, R.H. Cohen et al., "Very High Resolution Simulation of Compressible Turbulence on the Ibm-Sp System," Proc. SC99: High Performance Networking and Computing Conf. (electronic publication), 1999.

[16] W.H. Press, S.A. Teukolsky et al., Numerical Recipes in C: The Art of Scientific Computing, 2nd, rev. ed. Cambridge Cambridgeshire; New York: Cambridge University Press, 1997.

[17] D.A. Reed, R.A. Aydt et al., "An Overview of the Pablo Performance Analysis Environment," Department of Computer Science, University of Illinois, 1304 West Springfield Avenue, Urbana, IL 618011992.

[18] D.A. Reed, O.Y. Nickolayev et al., "Real-Time Statistical Clustering and for Event Trace Reduction," $J$. Supercomputing Applications and High-Performance Computing, 11(2):144-59, 1997.

[19] S. Shende, A.D. Malony et al., "Portable Profiling and Tracing for Parallel, Scientific Applications Using C++," Proc. SIGMETRICS Symp. Parallel and Distributed Tools (SPDT), 1998, pp. 134-45.

[20] M. Snir, S. Otto et al., Eds., Mpi--the Complete Reference, 2nd ed. Cambridge, MA: MIT Press, 1998.

[21] J. Stasko, J. Domingue et al., Eds., Software Visualization: Programming as a Multimedia Experience,. Cambridge, MA: MIT Press, 1998.

[22] J.S. Vetter, "Performance Analysis of Distributed Applications Using Automatic Classification of Communication Inefficiencies," Proc. ACM Int'l Conf. Supercomputing (ICS), 2000.

[23] J.S. Vetter and D. Reed, "Managing Performance Analysis with Dynamic Statistical Projection Pursuit," Proc. SC99: High Performance Networking and Computing Conf. (electronic publication), 1999.

[24] F. Wong, R. Martin et al., "Architectural Requirements and Scalability of the Nas Parallel Benchmarks," Proc. SC99: High Performance Networking and Computing Conf. (electronic publication), 1999. 